\title{
Ultimate Limit of Electron-Spin Precession upon Reflection in Ferromagnetic Films
}

\author{
A. Hallal, ${ }^{1}$ T. Berdot, ${ }^{1}$ P. Dey, ${ }^{1}$ L. Tati Bismaths, ${ }^{1}$ L. Joly, ${ }^{1}$ A. Bourzami, ${ }^{2}$ F. Scheurer, ${ }^{1}$ \\ H. Bulou, ${ }^{1}$ J. Henk, ${ }^{3}$ M. Alouani, ${ }^{1}$ and W. Weber ${ }^{1}$ \\ ${ }^{1}$ Institut de Physique et Chimie des Matériaux de Strasbourg, UMR 7504, UdS-CNRS, 23 rue du Loess, BP 43, \\ F-67034 Strasbourg Cedex 2, France \\ ${ }^{2}$ Département de Physique, Université Ferhat-Abbas, AL-19000 Sétif, Algeria \\ ${ }^{3}$ Max-Planck-Institut für Mikrostrukturphysik, Weinberg 2, D-06120 Halle, Germany
}

(Received 2 March 2011; published 16 August 2011)

\begin{abstract}
We report the discovery of $180^{\circ}$ electron-spin precession in spin-polarized electron-reflection experiments on Fe films on $\operatorname{Ag}(001)$, the largest possible precession angle in a single electron reflection. Both experiments as a function of Fe film thickness and $a b$ initio calculations show that the appearance of this ultimate spin precession depends with utmost sensitivity on the relaxation of the $\mathrm{Fe}$ surface layers during growth. Similar spin precession is also predicted for other ferromagnetic films.
\end{abstract}

PACS numbers: 75.70.Ak, 75.70.Rf

The conventional spin electronics based on spin valves and magnetic tunnel junctions does not yet use the phenomenon of electron-spin precession under the influence of an electric or a magnetic field. However, this spin precession could bring new degrees of freedom to spin electronics, as it has been proposed [1] and demonstrated with semiconductors [2]. This makes the study of the electron-spin propagation through a metal or a semiconductor one of the major new challenges in spin electronics. We emphasize that the electron-spin precession, as discussed here, originates from the same mechanism as the spin-torque effect for which the action of a spin-polarized current of sufficient density on the magnetization of a ferromagnetic layer is observed [3].

The precessional motion of the electron-spin can be induced by different means: (i) an electric field which is either external, as proposed by Datta and Das [1], or internal [4]; (ii) a magnetic field which is either externally applied [5] or the (intrinsic) exchange field of a ferromagnetic material [6]. To obtain a sizable precession by application of external magnetic or internal electric fields, the electrons have to travel very long distances (at least several $\mu \mathrm{m})$. In contrast, the exchange field in ferromagnets is several orders of magnitude larger than typical applied magnetic fields so that distances of a few atomic layers are sufficient to obtain sizable precession angles [6].

While the experiment of Weber et al. [6] was done in transmission, we consider here spin precession in reflection geometry. Intuitively, one might argue that the reflection mode is not very effective in inducing precessional spin motion, as the interaction time with the ferromagnetic material and, thus, with its exchange field is very short (in the fs range). However, it has been shown that spin precession upon reflection is possible [7-9].

In this Letter we show, by taking the example of Fe films on $\operatorname{Ag}(001)$, that the spin precession angle in reflection can indeed reach its maximum possible value of $180^{\circ}$. This marks the ultimate limit of electron-spin manipulation in reflection, that is spin reversal. Our $a b$ initio calculations provide strong evidence that the origin of this giant spin precession is the relaxation of the Fe lattice during growth on $\mathrm{Ag}$ substrate. Based on further calculations, we predict a similar behavior for fcc-Co(001), bcc-Co(001), and bcc$\mathrm{Ni}(001)$.

In the experiment (see Fig. 1), a GaAs-based electron source produces a spin-polarized electron beam of $70 \%$ polarization by means of optical pumping with circularly polarized light [10]. The polarized electron beam is incident at $45^{\circ}$ with respect to the sample surface normal with the in-plane projection of the wave vector along the [100] direction of the Fe film. The Fe film, having its magnetization in-plane for thicknesses above $0.6 \mathrm{~nm}$ [11], is remanently magnetized along the easy direction of magnetization, i.e., the [100] direction, by applying a magnetic field pulse of 500 Oe. The energy of the

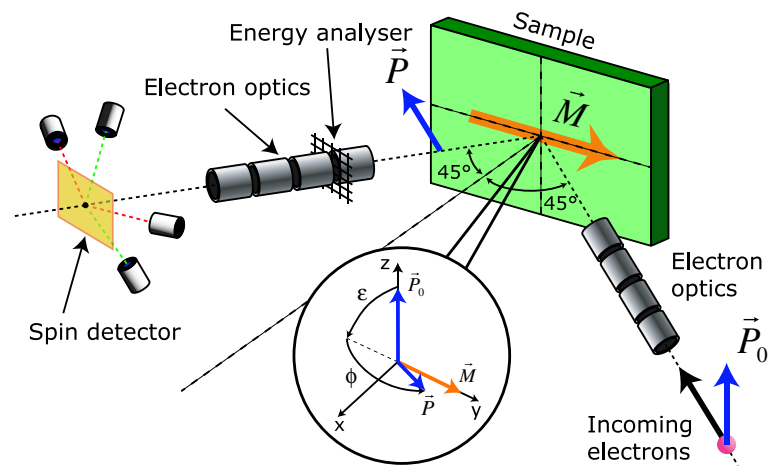

FIG. 1 (color online). Spin-polarized electron-reflection experiment, consisting of a spin-polarized electron source, a remanently magnetized sample, a retardation grid for the energy analysis, and a spin detection system. The two types of spin motion, i.e., the precession $\varepsilon$ and the rotation $\phi$, are defined in the inset. 
specularly reflected electrons is analyzed by a retarding grid analyzer. In the following we focus on the behavior of the elastically scattered electrons. The electrons are subsequently accelerated to an energy of $100 \mathrm{keV}$ to measure the transverse components of the spin polarization vector $\vec{P}$ via Mott scattering [12].

To obtain a maximum precessional spin motion of the reflected electrons it is crucial to orient the initial spin polarization $\vec{P}_{0}$ perpendicularly to the magnetization $\vec{M}$ of the ferromagnetic Fe film [6]. If we suppose for commodity a completely polarized electron beam, the spinor wave function of an incident electron is then a superposition of a majority- (magnetic moment parallel to $\vec{M}$ ) and a minority-spin wave function (magnetic moment antiparallel to $\vec{M})$ with equal weights: $\chi_{0} \propto(1,0)+(0,1)$. Because of the spin-dependent reflection at the ferromagnetic surface, these weights become different in magnitude and phase: $\chi \propto\left(\left|r^{\dagger}\right| e^{i \theta^{\dagger}},\left|r^{\downarrow}\right| e^{i \theta^{\downarrow}}\right)$ where $\left|r^{\dagger, \downarrow}\right|$ and $\theta^{\dagger, \downarrow}$ are, respectively, the moduli and the phases of the spindependent complex reflection amplitudes. This results in a precession of the spin polarization vector $\vec{P}$ by an angle $\varepsilon=\theta^{\downarrow}-\theta^{\uparrow}$ around $\vec{M}$ and in a rotation by an angle $\phi=\arctan \left(\left(\left|r^{\dagger}\right|^{2}-\left|r^{\downarrow}\right|^{2}\right) / 2\left|r^{\dagger} \| r^{\downarrow}\right|\right)$ in a direction either parallel or antiparallel to $\vec{M}$ (see inset in Fig. 1).

To understand the existence of the spin motion at a simple level we assume that the electrons within the ferromagnetic layer experience an exchange interaction which leads to an energy splitting of the two spin bands. In this simple model, electrons are scattered at the surface from a rectangular potential that has different heights for spin-up and spin-down electrons. This elementary problem can be solved exactly, and a spin motion appears in fact in this model [13]. However, this simple model is not realistic because it does not include the details of the electronic band structure. A realistic description requires the calculation of the spin-polarized low-energy electron diffraction from a $\mathrm{Fe}(001)$ surface as we did in this work.

$\operatorname{An} \operatorname{Ag}(001)$ single crystal is cleaned by Ar-ion sputtering and annealing up to $800 \mathrm{~K}$. Subsequently, Fe is deposited at room temperature from an Fe rod heated by electron beam bombardment. The growth of $\mathrm{Fe}$ on $\operatorname{Ag}(001)$ has been extensively investigated in the past, but gave rise to a controversy. Some experiments indicated a layer-by-layer growth [14-16], whereas others found island growth [17]. The key to understand this controversy might probably be different evaporation rates in the aforementioned experiments. While Fe films deposited at a "fast" rate of $0.2 \mathrm{~nm} / \mathrm{min}$ do not grow layer-by-layer, deposition at a "slow" rate of $0.02 \mathrm{~nm} / \mathrm{min}$ leads to layer-by-layer growth, as evidenced by Auger spectroscopy and electron-reflection experiments [18]. For all the following measurements, we have chosen the slow deposition rate.

In a first step we varied the Fe film thickness and recorded $\varepsilon$ at $E-E_{F}=7 \mathrm{eV}$ (inset in Fig. 2). In general, one would expect that $\varepsilon$ saturates for Fe thicknesses larger

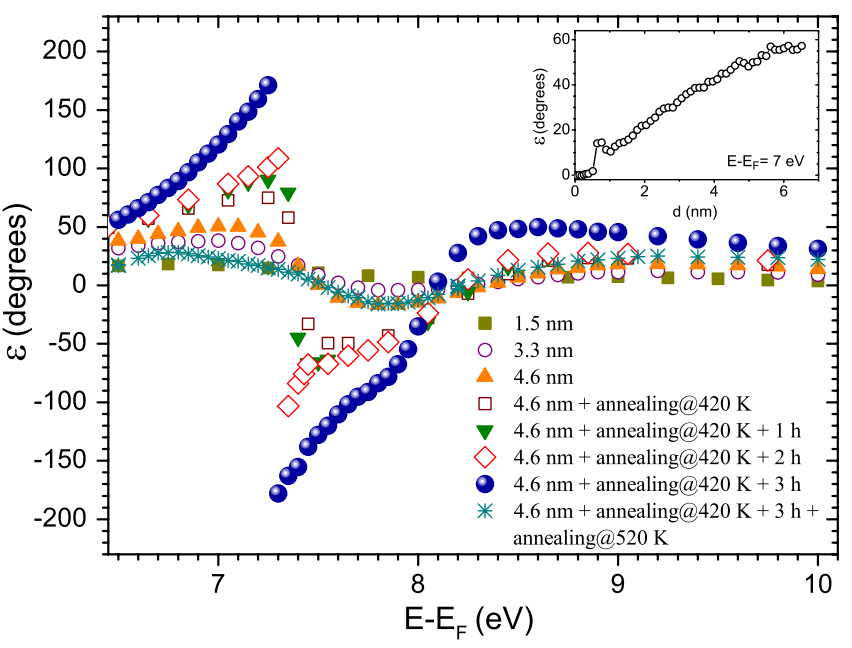

FIG. 2 (color online). Precession angle $\varepsilon$ versus primary electron energy $E-E_{F}$ for different Fe samples. The inset shows $\varepsilon$ versus Fe film thickness at a primary electron energy of $7 \mathrm{eV}$.

than the electron penetration depth (less than $1 \mathrm{~nm}$ ). Instead, we observed above $1 \mathrm{~nm}$ Fe thickness an almost linear increase of $\varepsilon$ which levels off only for thicknesses larger than $5 \mathrm{~nm}$. A similar behavior is also found for $\phi$ (not shown). We assume that changes of the electronic structure and hence of the spin-motion angles are due to variations of the Fe lattice parameter upon film growth. In fact, the bulk lattice parameters of bcc-Fe $(0.286 \mathrm{~nm})$ and fcc $\mathrm{Ag}(0.408 \mathrm{~nm})$ differ, resulting in a misfit of $-0.9 \%$. As a consequence, strain is established in the Fe film during its pseudomorphic growth on $\operatorname{Ag}(001)$, which is successively relieved for thicknesses above a critical value by the creation of interfacial dislocations in the film [19]. The lattice parameter of the film is thus expected to vary over a wide thickness range.

In a second step we recorded $\varepsilon$ versus energy for selected Fe samples (Fig. 2). Around $E-E_{F}=7.4 \mathrm{eV}$ a "plus or minus" structure becomes more pronounced with increasing thickness. Supposing that the increase of $\varepsilon$ with Fe thickness is due to strain relaxation, we expect a further increase by annealing the Fe film. Because higher temperatures promote strain relaxation, we annealed the $4.6 \mathrm{~nm}$ Fe film at $420 \mathrm{~K}$ for $10 \mathrm{~min}$ before measurement. As a result, we obtained a substantial increase of the $\varepsilon$ structure. Further measurements show that this feature is not yet stable but its amplitude increases further with time. This finding points clearly to the presence of a very slow relaxation process. After about $3 \mathrm{~h}$ the $\varepsilon$ structure stabilized and exhibited the maximum value of $180^{\circ}$. It is noted that a further increase of $\varepsilon$ above $180^{\circ}$ is indistinguishable from a rise of $\varepsilon$ from $-180^{\circ}$ towards less negative values. We emphasize that a thick Fe film annealed at $420 \mathrm{~K}$ for a longer time (30 $\mathrm{min}$ ) shows directly the $180^{\circ}$ structure without the need for additional waiting. Finally, the Fe sample showing the $180^{\circ}$ structure has been annealed 
at $520 \mathrm{~K}$ for $30 \mathrm{~min}$, leading to surface diffusion of $\mathrm{Ag}$, as evidenced by Auger spectroscopy. This results in a strong decrease of the $\varepsilon$ structure's amplitude.

Inspection of $\varepsilon$ and $\phi$ as well as the reflected intensity $I$ over a wider energy range (Fig. 3; $4.6 \mathrm{~nm}$ Fe film annealed at $420 \mathrm{~K}$ ) shows that the unique $\varepsilon$ structure at $7.4 \mathrm{eV}$ is accompanied by a pronounced minimum of $I$. The rotation angle $\phi$ shows also a strong structure in the energy range $7-8 \mathrm{eV}$ with a minimum value of $-60^{\circ}$. We emphasize that the maximum and minimum values are $\pm 90^{\circ}$ which corresponds to the electron-spin polarization being either parallel $(+)$ or antiparallel $(-)$ to the magnetization.

We now corroborate our hypothesis that the relaxation of the Fe lattice during growth is responsible for the ultimate electron-spin precession upon reflection, by means of $a b$ initio calculations of the electronic band structure of Fe films [20]. The electronic band structure is obtained using the full-potential linearized augmented plane wave (FLAPW) method [21] and the spin motion by the Korringa-Kohn-Rostoker (KKR) method [22]. In order to make a comparison with our experimental data, the geometry for the calculations was chosen identical to the experimental one. First, we inspected the spin-dependent band structure of $\mathrm{Fe}$ for various lattice relaxations (not shown) in order to evidence features that may change pronouncedly as a function of relaxation. However, no significant changes are found in the energy range of the giant spin precession. We note that the spin-down and -up reflection phases, whose difference determines $\varepsilon$, do not appear in the electronic band structure. It is only in the reflection process and thus in the KKR calculations in which the phases appear.

The KKR calculations clearly reproduce the experimental trends for both $\varepsilon$ and $\phi$, especially the $\varepsilon$ structure around $7.8 \mathrm{eV}$ for bcc-Fe(001) (Fig. 4, top, middle)

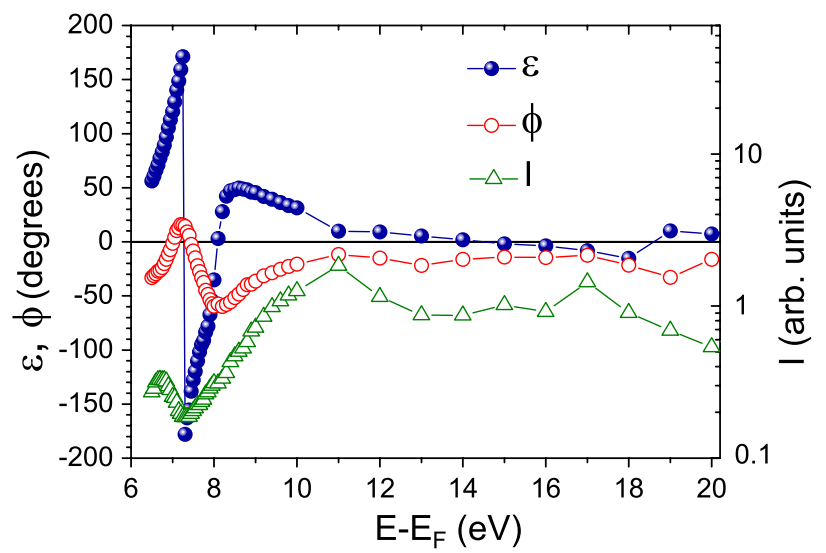

FIG. 3 (color online). Spin-integrated electron reflectivity $I$, precession angle $\varepsilon$ and rotation angle $\phi$ as a function of the primary electron energy $E-E_{F}$ for a $4.6 \mathrm{~nm}$ thick Fe film after annealing at $420 \mathrm{~K}$. The lines are guides to the eye. Note the logarithmic intensity scale. coincides with its experimental counterpart at $7.4 \mathrm{eV}$. For fcc-Co(001) (Fig. 4, top, left), bcc-Co(001) (not shown), and bcc-Ni(001) (Fig. 4, top, right), we predict similar structures around 3.4, 7.6, and $16.7 \mathrm{eV}$, respectively. Interestingly, the energy position at which the $\varepsilon$ structure appears varies very strongly with the ferromagnetic material. In contrast, for fcc-Fe(001) and fcc-Ni(001) we could not identify such a structure in our calculations.

For large Fe lattice strain the $\varepsilon$ structure is relatively small (Fig. 4, top, middle) but becomes more pronounced with decreasing strain. In particular, a very strong increase is found between $\Delta a_{\perp} / a_{\perp}^{\text {bulk }}=-0.8 \%$ and $-0.5 \%$, with $\Delta a_{\perp}=a_{\perp}^{\text {film }}-a_{\perp}^{\text {bulk }}$ being the difference between the out-of-plane lattice parameters of the film and bulk. For values in the range of $[-0.5 \%, 0.7 \%]$, the precession angle reaches the ultimate limit of $180^{\circ}$. In bcc-Co(001) (not shown) and bcc-Ni(001), the $180^{\circ}$-structure appears for relaxations in the range of $[-0.8 \%,-0.5 \%]$ and $[-1.5 \%,-1 \%]$, respectively. In fcc-Co(001) a $180^{\circ}$

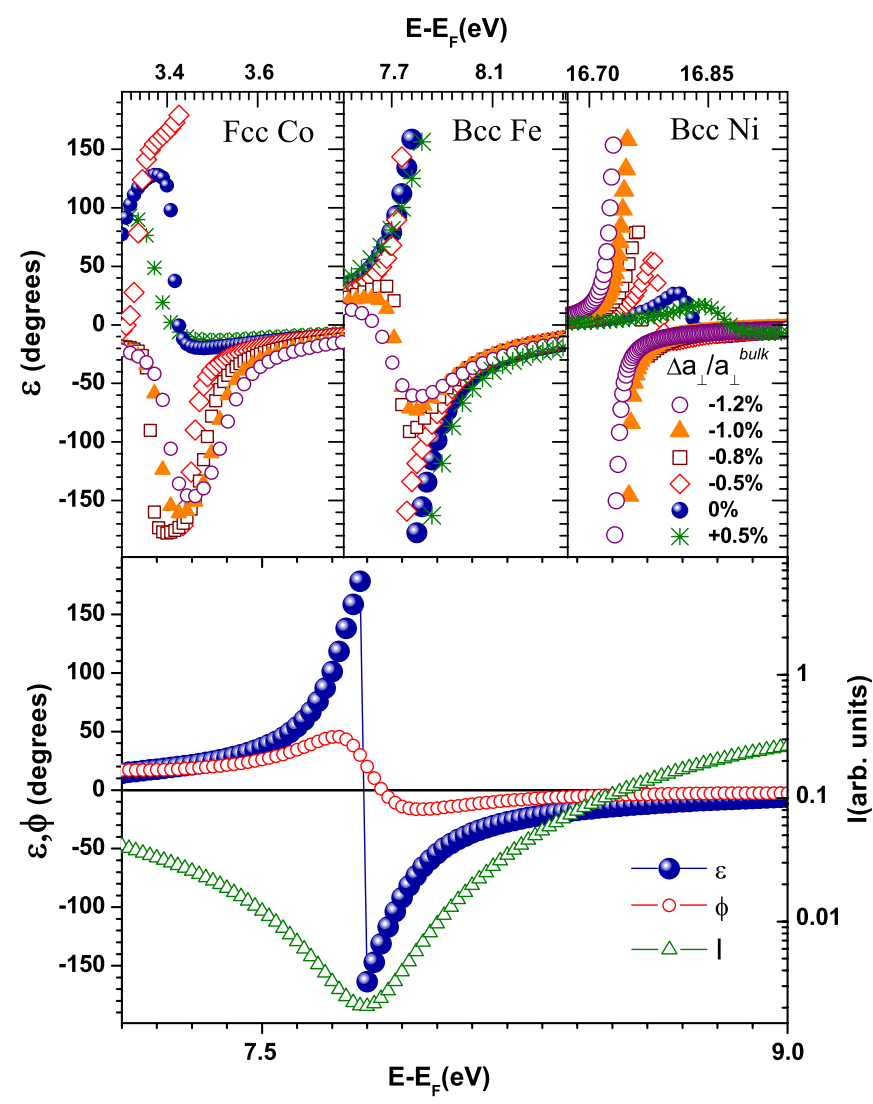

FIG. 4 (color online). Top: Calculated precession angle $\varepsilon$ versus electron energy for different degrees of lattice relaxation for fcc-Co(001) (left), bcc-Fe(001) (middle), and bcc-Ni(001) (right). We note that the corresponding calculated vacuum levels are at 3.3, 2.8, and $5.5 \mathrm{eV}$, respectively. Bottom: Calculated precession angle $\varepsilon$, rotation angle $\phi$, and reflected intensity $I$ versus electron energy for a completely relaxed (not strained) $\mathrm{Fe}$ film. Note the logarithmic intensity scale. 
structure appears around $-0.5 \%$ relaxation. Interestingly, it develops into $\mathrm{a}-180^{\circ}$ peak for stronger strained lattices before its amplitude decreases again. Thus, in any case, the $\varepsilon$ structure is extremely sensitive to the lattice relaxation.

For a completely relaxed (not strained) Fe film, i.e., $\Delta a_{\perp} / a_{\perp}^{\text {bulk }}=0$, the $\varepsilon$ structure is accompanied by a strong structure in $\phi$ and a pronounced minimum in $I$ (Fig. 4, bottom), as in the experiment (Fig. 3). We note that although the experimental and the calculated $\phi$ structures are vertically shifted by about $40^{\circ}$ with respect to each other, their amplitudes are comparable (experiment: $76^{\circ}$, calculation: $62^{\circ}$ ). We note as well that the $\varepsilon$, the $\phi$, and the $I$ structures are considerably sharper than in the experiment. Since this finding cannot be explained by the energy resolution of the experimental setup $(\sim 0.3 \mathrm{eV})$, we can only speculate on the reason for this difference.

How can one understand in simple terms that a giant spin precession appears at a particular electron energy and is accompanied by an intensity minimum? To obtain a strong $\varepsilon$ structure it is clear that the spin-dependent phases $\theta^{\uparrow, \downarrow}$ have to exhibit a strong variation, a behavior that is reminiscent of the Ramsauer-Townsend effect. In the $1920 \mathrm{~s}$ Ramsauer and Townsend observed that the scattering of very slow electrons ( $0.7 \mathrm{eV}$ kinetic energy) by Ar-atoms was much weaker than expected from gas kinetic theory [23]. It was Bohr who suggested that one is dealing here with a resonance phenomenon which combines weak scattering with a strong change of the scattering phase. In fact, in order to get a minimum scattering intensity the wave function of the electrons within the scattering potential has to fulfill certain boundary conditions which in turn lead to a certain resonance condition for the electron energy. For example, considering a simple square potential well of width $a$ and depth $U$, the resonance condition reads: $k a=\frac{2 n+1}{2} \pi$ with $k \sim \sqrt{E_{\text {kin }}+U}$ the electron momentum inside the potential well, $E_{\mathrm{kin}}$ the kinetic energy of the electrons outside the potential well and $n$ an integer. On the other hand, the scattering phase is known to change quite strongly around the resonance energy, because the phase matching condition between scattered and unscattered wave is not anymore fulfilled for off-resonance energies. As the resonance phenomenon appears for the majority-spin and the minority-spin wave at different energies separated by the exchange splitting the precession angle $\varepsilon=\theta^{\downarrow}-\theta^{\uparrow}$ must necessarily exhibit a strong change. We emphasize, however, that the situation in a crystal, which is the case here, is much more complex and requires a multiple-scattering approach to obtain realistic results. This is the mean reason for a full KKR calculation of the spin motion.

The strong sensitivity of the giant spin precession structure on little changes of the lattice parameter becomes plausible when we observe the behavior of both $\varepsilon$ and $I$. Both experiment and theory show that the strength of the $\varepsilon$ structure is related to the strength of the intensity minimum, i.e., the more pronounced the intensity minimum the more pronounced the $\varepsilon$ structure (not shown). It is now important to understand that for small intensities $I^{\uparrow, \downarrow}\left(=\left|r^{\uparrow, \downarrow}\right|^{2}\right)$ a given change of $r^{\uparrow, \downarrow}$ leads to a much stronger variation of $\theta^{\uparrow, \downarrow}$ and thus in $\varepsilon$, than in the case where the intensities are larger.

In conclusion, spin-polarized electron-reflection experiments on carefully prepared Fe films on $\operatorname{Ag}(001)$ show that the spin precession angle in reflection can reach its maximum possible value, namely, $180^{\circ}$. This marks the ultimate limit of spin manipulation in reflection. Our ab initio calculations strongly support our hypothesis that the relaxation of the Fe lattice during growth is responsible for this effect. We hope that our work will motivate further studies to show, in particular, how the appearance of the strong $\varepsilon$-structure and its energy position could be further modified in a ferromagnetic film by the choice of the substrate, its orientation or by alloying.

M. A. and W. W. acknowledge support from ANR grants No. ANR-06-NANO-053-01 and No. ANR-09-BLAN0076-03, respectively. We acknowledge useful advice from D. Sébilleau.

[1] S. Datta and D. Das, Appl. Phys. Lett. 56, 665 (1990).

[2] B. Huang, D. J. Monsma, and I. Appelbaum, Phys. Rev. Lett. 99, 177209 (2007).

[3] E. B. Myers, D. C. Ralph, J. A. Katine, R. N. Louie, and R. A. Buhrman, Science 285, 867 (1999).

[4] Y. Kato, R.C. Meyers, A.C. Gossard, and D. D. Awschalom, Nature (London) 427, 50 (2004).

[5] J. M. Kikkawa and D.D. Awschalom, Nature (London) 397, 139 (1999).

[6] W. Weber, S. Riesen, and H. C. Siegmann, Science 291, 1015 (2001).

[7] L. Joly, J. K. Ha, M. Alouani, J. Kortus, and W. Weber, Phys. Rev. Lett. 96, 137206 (2006).

[8] L. Joly, L. Tati Bismaths, and W. Weber, Phys. Rev. Lett. 97, 187404 (2006).

[9] L. Joly, L. Tati Bismaths, F. Scheurer, and W. Weber, Phys. Rev. B 76, 104415 (2007).

[10] D. T. Pierce, R. J. Celotta, G.-C. Wang, W. N. Unertl, A. Galejs, C.E. Kuyatt, and S.R. Mielczarek, Rev. Sci. Instrum. 51, 478 (1980) and references therein.

[11] M. Stampanoni, A. Vaterlaus, M. Aeschlimann, and F. Meier, Phys. Rev. Lett. 59, 2483 (1987).

[12] J. Kessler, in Polarized Electrons (Springer, Berlin, 1985), chap. 8.

[13] M.D. Stiles and A. Zangwill, Phys. Rev. B 66, 014407 (2002).

[14] G. C. Smith, H. A. Padmore, and C. Norris, Surf. Sci. 119, L287 (1982).

[15] B. Heinrich, S. T. Purcell, J. R. Dutcher, K. B. Urquhart, J. F. Cochran, and A.S. Arrott, Phys. Rev. B 38, 12879 (1988).

[16] P. J. Schurer, Z. Celinski, and B. Heinrich, Phys. Rev. B 51, 2506 (1995). 
[17] H. Li, Y. S. Li, J. Quinn, D. Tian, J. Sokolov, F. Jona, and P. M. Marcus, Phys. Rev. B 42, 9195 (1990).

[18] L. Tati Bismaths, L. Joly, A. Bourzami, F. Scheurer, and W. Weber, Phys. Rev. B 77, 220405(R) (2008).

[19] A. Chambers and D.C. Jackson, Philos. Mag. 31, 1357 (1975).

[20] The volume of the lattice unit cell is assumed to be conserved, which is supported by LEED experiments on a similar system $(\mathrm{Fe} / \mathrm{GaAs}(001)$ [see T. Kebe et al., J. Phys. Condens. Matter 18, 8791 (2006)].

[21] J. Grotendorst, S. Blügel, and D. Marx, NIC Series 31, 85 (2006), http://www2.fz-juelich.de/nic-series/volume31/ bluegel.pdf.

[22] J. Henk, A. M. N. Niklasson, and B. Johansson, Phys. Rev. B 59, 13986 (1999) and references therein.

[23] C. Ramsauer, Ann. Phys. (Leipzig) 369, 513 (1921); V. A. Bailey and J. S. Townsend, Philos. Mag. 42, 873 (1921). 